\title{
Modified Approach for Feature Space Improvement of Imagery
}

\author{
Dr. Sandeep V. Rode $^{1}$ \\ Professor, Department of Electronics and Telecommunication, Sipna College of Engineering and Technology, \\ Amravati, India ${ }^{1}$
}

\begin{abstract}
This paper addresses the technique which is based on the maximum feature space improvement for the detailed analysis of the plant leaf images, implementation of the super-resolution to the given plant leaf image is possible by the method of locally linear embedding ( $L L E)$. In LLE, the feature space can be improved by imposing the neighbor pixels into feature space.
\end{abstract}

Keywords: Super Resolution, Plant Leaf, LLE

\section{INTRODUCTION}

Any plant leaf caries more information of the related diseases, if it is abnormal. Normal plant leaf doesn't shows any significant information related to diseases present on it. The most important part of leaf image is that, during processing we need to reduce the dimensions of the image. As the dimension tends to decreasing, the information of the image is going to reduce, to preserve the same information during the feature extraction process not exactly but theoretically exact information. The pattern can be improved by giving training to low resolution images by high resolution images [1]. The super resolution technique has numerous applications in the field of computer vision and machine learning. To improve the efficiency of processing algorithms for disease recognition, it is important to have the image super resolution to faithfully regenerate the patterns from training set.

\section{LITERATURE REVIEW}

The massive work is already done for super resolution. Our way in this paper is how the LLE method is beneficial for image feature space improvement, and we will discuss the work already done in this area, not in detailed but significant work. Paper by William Freeman, entailed as 'Example based Super resolution' [2], added it is possible to preserve the features by following steps i) Prediction, which includes the important frequencies, which is predicted by missing frequencies from given image. ii) Search Algorithm, is used to predict absolute best match from the training space. Michael Elad, suggest another paper which entitled as 'A fast Super-Resolution Reconstruction Algorithm for Pure Translational motion and Common Space- Invariant Blur' [3], the paper addresses the way to obtain the super resolution of given blurred image, the various algorithms were in order to complete this task, such as Maximum Likelihood, the efficiency and the computational cost will reduced using this algorithm. Paper by Kwang In Kim suggest [4], Kernel Hebbian Super resolution technique, the knowledge about high resolution which is required is obtained from KPCA (Kernel Principle Component
Analysis Method). Here KPCA is act upon the training space. Using the KPCA iterative steps the super resolution of the image is achieved called as Kernel Hebbian super resolution technique. The Bayesian Method of super Resolution, by Lyndsey [5], knowledge of distribution in order to formulate the probabilistic super resolution image. The paper by Hojatollah Yeganeh [6] used objective quality assessment of the given image, so this technique is more beneficial for very low resolution images. Here image interpolation technique is used to increase the feature space. The interpolation values were predicted from natural statistics of given image. In this paper we suggest the flexible and easy way to solve the super resolution problems for the given plant leaf image by Local Linear Embedding.

\section{LOCAL LINEAR EMBEDDING}

The LLE is based upon the pixel geometry interpretation, which is characterized as suppose the given image have $N$ real valued vectors say $\bar{X}_{i}$, provided that dimension is $D$. Here we have the neighbouring pixels which are linearly space with each other. In that case the Euclidean Space Cost Function (1) is formulated. The reconstruction related errors are measured by this cost function.

$$
\varepsilon(\mathrm{W})=\sum_{i}\left|\vec{X}_{i}-\sum_{j} W_{i j} \vec{X}_{j}\right|^{2}
$$

We can also represent $\varepsilon(\mathrm{W})$ as $\varepsilon^{q}$, which is when minimizing subject to constraint based least square problem.

Above eq. (1) can be re- written as (2),

$$
\varepsilon^{q}=\left|X_{t}^{q}-\sum_{x_{p}^{p} s \eta_{q}} \omega_{q} X_{s}^{p}\right|^{2}
$$

Now local gram matrix is $G_{q}$ for $X_{t}^{q}$ is given as

$$
G_{q}=\left(\mathrm{x}_{t}^{q} 1^{T}-X\right)^{T}\left(\mathrm{x}_{t}^{q} 1^{T}-X\right)^{T}
$$


From eq. (1), 1 is called column vector, and $X$ is $D x K$ matrix, whose columns being neighbour of $\mathrm{X}_{t}^{q}$. Now each subscript p can be formulated from $K$ dimensional vector $\omega_{q}$, so the following close loop solution is applied for least square problem (constrained). Its solution is given as shown below in eq. (4)

$$
w_{p}=\frac{G_{q}^{-1} 1}{1^{t} G_{q}^{-1} 1}
$$

By solving the linear system of equation for eq. (4), we get following analysis as given in eq. (5)

$$
G_{q} w_{q}=1
$$

And after normalizing the weights we will get,

$$
\sum_{x_{i}^{p} \varepsilon \eta_{\hat{q}}} \omega_{p q}=1
$$

So for all patches say $N_{t}$ in $x_{t}$, the weight matrix (reconstructed) as eq. (7)

$$
W=\left[\omega_{q p}\right] N_{t} N_{s}
$$

So, computing high resolution embedding as in eq. (8)

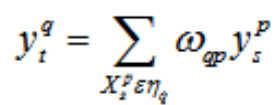

\section{FEATURE SPACE REPRESENTATION}

Color Images are represented as RGB space, so here we need to convert this RGB color space to the YIQ model. In YIQ color model, Y - represents luminance and rest of I and $\mathrm{Q}$ represents chrominance. So conversion from RGB to YIQ color model can be approximated by linear transformation. Here we $\mathrm{Y}$ channel is define features, which is going to exactly learn for feature space improvement, here we are going to keep I and Q channel as it is to that of low resolution plant leaf image. Also for low resolution image we are going to implement the first order gradient which could be alternative method, here let us consider fig. 1 , and chose center pixel as luminance parameter, then in this case, first gradient denoted as $\nabla Z_{25}$, and second order gradient vector is denoted as $\nabla^{2} Z_{25}$.which is derived for $7 \times 7$ local neighbours as given below,

$$
\nabla Z_{25}=\left[\begin{array}{c}
\left(Z_{26}-Z_{25}\right)+\left(Z_{25}-Z_{24}\right) \\
\left(Z_{32}-Z_{25}\right)+\left(Z_{25}-Z_{18}\right)
\end{array}\right]
$$

$$
\nabla Z_{25}=\left[\begin{array}{c}
\left(Z_{26}-Z_{24}\right) \\
\left(Z_{32}-Z_{18}\right)
\end{array}\right]
$$

\begin{tabular}{|l|l|l|l|l|l|l|}
\hline$Z_{1}$ & $Z_{2}$ & $Z_{3}$ & $Z_{4}$ & $Z_{5}$ & $Z_{6}$ & $Z_{7}$ \\
\hline$Z_{8}$ & $Z_{9}$ & $Z_{10}$ & $Z_{11}$ & $Z_{12}$ & $Z_{13}$ & $Z_{14}$ \\
\hline$Z_{15}$ & $Z_{16}$ & $Z_{17}$ & $Z_{18}$ & $Z_{19}$ & $Z_{20}$ & $Z_{21}$ \\
\hline$Z_{22}$ & $Z_{23}$ & $Z_{24}$ & $Z_{25}$ & $Z_{26}$ & $Z_{27}$ & $Z_{28}$ \\
\hline$Z_{29}$ & $Z_{30}$ & $Z_{31}$ & $Z_{32}$ & $Z_{33}$ & $Z_{34}$ & $Z_{35}$ \\
\hline$Z_{36}$ & $Z_{37}$ & $Z_{38}$ & $Z_{39}$ & $Z_{40}$ & $Z_{41}$ & $Z_{42}$ \\
\hline$Z_{43}$ & $Z_{44}$ & $Z_{45}$ & $Z_{46}$ & $Z_{47}$ & $Z_{48}$ & $Z_{49}$ \\
\hline
\end{tabular}

Fig. 1: A 7x7 local neighbourhood for in the low resolution image.

Computing second order gradient from fig. 1, as given in eq. (10),

$$
\begin{aligned}
& \nabla^{2} Z_{25}=\left[\begin{array}{c}
\left(Z_{28}-Z_{25}\right)+\left(Z_{25}-Z_{22}\right) \\
\left(Z_{46}-Z_{25}\right)+\left(Z_{25}-Z_{4}\right)
\end{array}\right] \\
& \nabla^{2} Z_{25}=\left[\begin{array}{c}
\left(Z_{28}-2 Z_{25}+Z_{22}\right) \\
\left(Z_{46}-2 Z_{25}+Z_{4}\right)
\end{array}\right]
\end{aligned}
$$

\section{Conclusion}

$\mathrm{Km}$ By studying and experimenting several gradient masks based on resolution and we are formulating $7 \mathrm{x}$ resolution model, i.e. improvement in feature space to study the significant feature space in plant leaf by LLE algorithm is possible, which is discussed under following figure, and context.

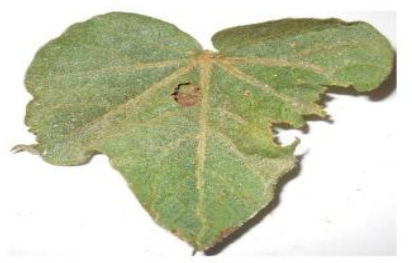

Fig. 2: Training low-resolution source

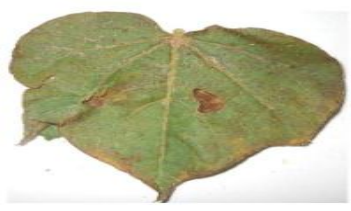

Fig 3: Training sample of high resolution source. 


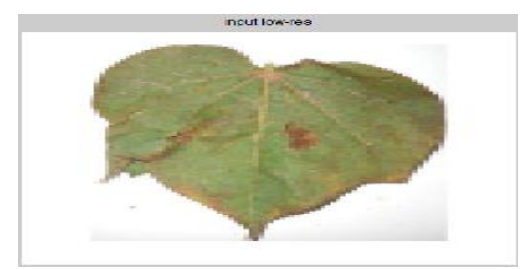

Fig. 4: Input Low Resolution Image

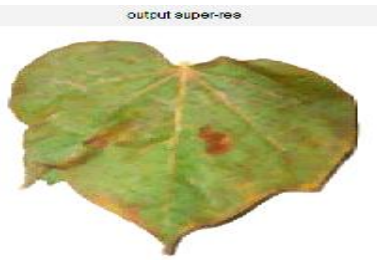

Fig 5: Output Super Resolution Image

In this paper we successfully implement LLE model of super resolution in order to expand feature space for studying diseases pattern on the plant leaf. A very important thing of LLE is that it is generalize for super resolution to increase the feature space. The training to low resolution image (fig 4) is estimated from fig. 2 and fig. 3 which produce more significant feature space image (fig 5). Here first order and second order gradients as given in eq. $[9,10]$ performs significant roll to improve intensity changes and satisfy the smoothness constraints of resulting image( fig.5).

\section{ACKNOWLEDGMENT}

I am thankful to lab facilities provided by Sipna College of Engineering and Technology, Amravati to conduct this research work successfully.

\section{REFERENCES}

[1] Hong Chang, Dit-Yan Yeung, Yimin Xiong, 'Super-Resolution Through Neighbor Embedding', IEEE Computer Society Conference on Computer Vision and Pattern Recognition, June- 27, 2004,July 2, 2004

[2] William T. Freeman, Thouis R. Jones, and Egon C. Pasztor,'Example Based Super Resolution.', IEEE Computer Graphics and Applications/March/April 2002.

[3] Michael Elad, and Yacov Hel-Or, 'A Fast SuperResolutionReconstruction Algorithm for Pure Translational Motion and Common Space-Invariant Blur', IEEE TRANSACTIONS ON IMAGE PROCESSING, VOL. 10, NO. 8, AUGUST 2001.

[4] Kwang In Kim, Matthias O. Franz, and Bernhard Sch"olkopf, 'Kernel Hebbian Algorithm for Single-Frame Super-Resolution', Statistical Learning in Computer Vision, ECCV 2004 Workshop, Prague, Czech Republic, May 2004, pp. 135-149.

[5] Lyndsey C. Pickup, David P. Capel, Stephen J. Roberts and Andrew Zisserman, 'Bayesian Methods For Image Super-Resolution', The Computer Journal Vol. 00 No. 0, 2006.

\section{BIOGRAPHY}

Sandeep V. Rode received master degree in 2004 and $\mathrm{PhD}$ in 2013 in Electronics Engineering from Sant Gadge baba Amravati University, Amravati, India. He has 17 years of teaching experience and presently working as Professor in Department of Electronics and telecommunication at Sipna college of Engineering and
Technology, Amravati (India). His main research interest includes signal processing, image processing Electronics circuit design, logic etc. He is a member of IEEE, IETE, IE and ISTE. He has published 29 papers in National and International Journals and conferences. 